\title{
Refining "Long-COVID" by a Prospective Multimodal Evaluation of Patients with Long-Term Symptoms Attributed to SARS-CoV-2 Infection
}

\author{
Marc Scherlinger (D) · Renaud Felten · Floriane Gallais • Charlotte Nazon • Emmanuel Chatelus · Luc Pijnenburg • \\ Amaury Mengin · Adrien Gras · Pierre Vidailhet · Rachel Arnould-Michel · Sabrina Bibi-Triki · Raphaël Carapito · \\ Sophie Trouillet-Assant - Magali Perret • Alexandre Belot · Seiamak Bahram • Laurent Arnaud • Jacques- \\ Eric Gottenberg · Samira Fafi-Kremer · Jean Sibilia
}

Received: April 27, 2021 / Accepted: June 15, 2021 / Published online: July 10, 2021

(C) The Author(s) 2021

\section{ABSTRACT}

Introduction: COVID-19 long-haulers, also decribed as having "long-COVID" or post-acute COVID-19 syndrome, represent $10 \%$ of COVID19 patients and remain understudied.

Marc Scherlinger, Renaud Felten, Floriane Gallais, Samira Fafi-Kremer and Jean Sibilia participated equally in the study.

Supplementary Information The online version contains supplementary material available at https:// doi.org/10.1007/s40121-021-00484-w.

M. Scherlinger $(\bowtie) \cdot$ R. Felten · E. Chatelus · L. Pijnenburg · R. Arnould-Michel · L. Arnaud . J.-E. Gottenberg · J. Sibilia $(\bowtie)$

Rheumatology Department, Centre Hospitalier Universitaire de Strasbourg, 1 Avenue Molière, 67098 Strasbourg, France

e-mail: marc.scherlinger@chru-strasbourg.frJ. Sibilia e-mail: jean.sibilia@chru-strasbourg.fr

M. Scherlinger · R. Felten · E. Chatelus . L. Pijnenburg · R. Arnould-Michel · L. Arnaud . J.-E. Gottenberg · J. Sibilia Centre National de Référence Des Maladies AutoImmunes Et Systémiques Rares, Est/Sud-Ouest (RESO), Service de Rhumatologie du CHU de Strasbourg, 1 Avenue Molière, 67200 Strasbourg Cedex, France

F. Gallais · C. Nazon · S. Fafi-Kremer Virology Diagnostic Laboratory, Centre Hospitalier Universitaire de Strasbourg, 3 Rue Koeberlé, 67000 Strasbourg, France
Methods: In this prospective study, we recruited 30 consecutive patients seeking medical help for persistent symptoms (>30 days) attributed to COVID-19. All reported a viral illness compatible with COVID-19. The patients underwent a multi-modal evaluation, including clinical, psychologic, virologic and specific immunologic assays and were followed longitudinally. A group of 17 convalescent COVID19 individuals without persistent symptoms were included as a comparison group.

Results: The median age was 40 [interquartile range: $35-54]$ years and 18 (60\%) were female.

A. Mengin - A. Gras · P. Vidailhet

Centre Hospitalier Universitaire de Strasbourg, 1 Avenue Molière, 67098 Strasbourg, France

\section{S. Bibi-Triki · R. Carapito - S. Bahram · L. Arnaud . S. Fafi-Kremer $\cdot$ J. Sibilia Laboratoire D'ImmunoRhumatologie Moléculaire, Institut National de La Santé Et de La Recherche Médicale (INSERM) UMR_S 1109, Institut Thématique Interdisciplinaire (ITI) de Médecine de Précision de Strasbourg, Transplantex NG, Faculté de Médecine, Fédération Hospitalo-Universitaire OMICARE, Fédération de Médecine Translationnelle de Strasbourg (FMTS), Université de Strasbourg, Strasbourg, France}

S. Trouillet-Assant · M. Perret · A. Belot National Reference Centre for Rare Rheumatic and AutoImmune Diseases in childrEn RAISE, Hospices Civils de Lyon, CIRI, Centre International de Recherche en Infectiologie, Inserm, U1111, Université Claude Bernard Lyon 1, CNRS, UMR5308, ENS de Lyon, 69007 Lyon, France 
At a median time of 152 [102-164] days after symptom onset, fever, cough and dyspnea were less frequently reported compared with the initial presentation, but paresthesia and burning pain emerged in 18 (60\%) and 13 (43\%) patients, respectively. The clinical examination was unremarkable in all patients, although the median fatigue and pain visual analog scales were 7 [5-8] and 5 [2-6], respectively. Extensive biologic studies were unremarkable, and multiplex cytokines and ultra-sensitive interferon- $\alpha 2$ measurements were similar between long-haulers and convalescent COVID-19 individuals without persistent symptoms. Using SARS-CoV2 serology and IFN- $\gamma$ ELISPOT, we found evidence of a previous SARS-CoV-2 infection in $50 \%(15 / 30)$ of patients, with evidence of a lack of immune response, or a waning immune response, in two patients. Finally, psychiatric evaluation showed that $11(36.7 \%), 13(43.3 \%)$ and $9(30 \%)$ patients had a positive screening for anxiety, depression and post-traumatic stress disorder, respectively.

Conclusions: Half of patients seeking medical help for post-acute COVID-19 syndrome lack SARS-CoV-2 immunity. The presence of SARSCoV-2 immunity, or not, had no consequence on the clinical or biologic characteristics of post-acute COVID-19 syndrome patients, all of whom reported severe fatigue, altered quality of life and psychologic distress.

Keywords: Disability; Long-COVID; Pain; Patient perspective; SARS-CoV-2

J.-E. Gottenberg

IBMC, UPR3572, CNRS, Strasbourg, France

\section{Key Summary Points}

Why carry out this study?

Long-COVID, or post-acute COVID-19 syndrome, has been reported to occur in up to $10 \%$ of all COVID-19 patients.

Little information is available on the clinical characteristics and pathophysiology of this syndrome. In the present study, we aim to describe these characteristics and to investigate potential underlying mechanisms.

What was learned from this study

Among 30 consecutive patients reporting persistent symptoms (median 6 months) self-attributed to COVID-19, pain, fatigue and disability were reported in virtually all patients.

More than one third of patients suffer from psychologic disorders such as anxiety, depression and/or post-traumatic stress disorder, regardless of SARS-CoV-2 immunity.

At the time of evaluation, only $50 \%$ of patients had cellular and/or humoral signs of a past SARS-CoV-2, and serology positivity varied depending on the kit used.

Exhaustive clinical, biologica and immunologic evaluations failed to find an alternative diagnosis or to identify a specific cytokine signature including type I interferon.

\section{DIGITAL FEATURES}

This article is published with digital features, including a summary slide, to facilitate understanding of the article. To view digital features for this article go to https://doi.org/10.6084/ m9.figshare.14786100. 


\section{INTRODUCTION}

After SARS-CoV-2 infection, some patients may continue to experience symptoms for several months [1], a condition which has been termed "long-COVID," "COVID-19 long-haulers," or post-acute COVID-19 syndrome [2]. These longterm symptoms contribute to the worldwide COVID-19 burden and have been extensively communicated by patients and physicians $[3,4]$. Manifestations of post-acute COVID-19 syndrome are clinically diverse, and multiple underlying mechanisms are likely to be implicated. Patients who underwent extensive hospitalization due to severe disease may exhibit chronic lung or heart injury due to the heightened immune response $[5,6]$, micro- or macrovascular thrombotic neurologic complications [7] and/or physical deconditioning [8]. Indeed, a recent cohort study found that among 1733 hospitalized COVID-19 patients, 76\% reported persistence of at least one symptom after 6 months, and objective pulmonary abnormalities were found in $>20 \%$ [9]. However, these pathologic mechanisms are less likely to explain the manifestations in patients with initially non-severe COVID-19 who go on to experience long-lasting symptoms. Among those plausible mechanisms, viral-induced autoimmunity should be investigated in light of the accumulating data reporting this phenomenon during or following acute COVID-19 [10]. Although 10\% of COVID-19 patients may show chronic symptoms ( $>12$ weeks) [11], data on the clinical presentation, biologic characteristics and overall prognosis of such patients are scarce $[9,12]$. Moreover, the virologic definition of post-acute COVID-19 syndrome in terms of serology and other specific immunologic assays remains to be precisely established $[12,13]$.

The aim of our study was to describe the clinical and biologic characteristics of postacute COVID-19 syndrome by conducting a multimodal evaluation of consecutive patients seeking medical help for persistent symptoms attributed to COVID-19 and to compare their characteristics to convalescent COVID-19 individuals without persistent symptoms. This evaluation also aimed to investigate the potential underlying mechanisms, including autoimmunity and psychologic distress.

\section{METHODS}

\section{Patients and Ethical Considerations}

We screened 34 consecutive patients seeking medical help for persistent symptoms attributed to COVID-19 for participation in this systematic, prospective study. Patients were recruited in the "Grand-Est" region of France, the area with the highest incidence of SARS-CoV-2 infection during the first epidemic wave in France (February to April 2020) [14]. Information about a post-acute COVID-19 syndrome consultation in our tertiary center was advertised through local media and social networks, and interested patients were then asked to participate in the study. All patients provided written informed consent. The data from 17 age-matched patients with a history of nonsevere COVID-19 (confirmed with RT-PCR or serology) at least 3 months before immuno-virologic assays, and without persistent symptoms, were included as controls (these patients were initially recruited as part of the Study "SeroCOVHUS," ClinicalTrials.gov Identifier: NCT04441684, Ethical Committee authorization from SUD MEDITERRANEE III, No. 2020.04.15 bis_ 20.04.10.66856). The sera from 5 patients with acute severe COVID-19 (hospitalized in the intensive care unit) and from 18 patients with active systemic lupus erythematosus were used as a positive control for multiplex cytokine and interferon- $\alpha 2$ measurements, respectively. The present study is part of the "COVID-HUS" study, which was approved by the Ethics Committee of the University Hospital of Strasbourg (NCE-2020-51).

\section{Medical Evaluation}

All patients were evaluated at our reference center for rare systemic and autoimmune diseases. Five physicians (MS, RF, EC, LP and JS) conducted the clinical evaluation with a 
physical examination and a standardized questionnaire on medical and SARS-CoV-2-related infection history. Dedicated questionnaires were used to evaluate subjective symptoms: DN4 for neuropathic pain [15], Fibromyalgia Rapid Screening Tool (FiRST [16]) and visual analog scales for fatigue/pain/dryness. Additionally, patients underwent systematic blood testing to rule out alternative diagnoses (full list in Supplementary File 1).

\section{Virologic and Immunologic Evaluation}

As a second step to the clinical evaluation, immuno-virologic assays were conducted. Testing included an extensive serology evaluation using four commercial assays: a lateral flow assay testing for the receptor-binding domain (RBD) of the spike protein as an antigenic source (Biosynex BSS IgM/IgG assay); two enzyme-linked immunosorbent assays (ELISA) targeting the RBD (Wantai total antibody assay) and the S1 domain (Euroimmun IgG assay); a third ELISA testing for anti-nucleocapsid (antiN) IgG (Abbott Architect IgG). To explore the SARS-CoV-2-specific T-cell response, heparinanticoagulated blood tubes were collected for interferon gamma (IFN- $\gamma$ ) enzyme-linked immunospot (ELISPOT) assay. Detailed methods of SARS-CoV-2 IFN- $\gamma$ ELISPOT are described in Supplementary File 2. To investigate the presence of remnant SARS-CoV-2 RNA, nasopharyngeal and stool RT-PCR were conducted in patients who accepted to take the test (methods in Supplementary File 2). To complete the immunologic evaluation, interferon- $\alpha 2$ (IFN- $\alpha 2$ ), with Single Molecule Array (SIMOA, Quanterix [17]) and cytokines (interleukin $1 \beta$ [IL-1ß], IL-2, IL-4, IL-5, IL-6, IL-8, IL-10, IL12p70, IL-13, IL-17A, IL18, IFN- $\gamma$, IP-10, G-CSF, GM-CSF, MCP-1, MIP- $1 \alpha$, MIP- $1 \beta$ and TNF- $\alpha$, using a custom multiplex cytokine assay; Luminex Thermo Fisher) were measured from serum samples.

\section{Psychologic Evaluation}

A standardized interview was conducted by a clinical psychologist (R.A.-M.). The interview aimed at evaluating psychiatric history and treatments, presence of sleep issues and increase of anxiolytic use. The impact of the COVID-19 pandemic on quality of life was assessed by using the Medical Outcomes Study 36-item Short-form (SF-36) and Health Assessment Questionnaire (HAQ). Patients' perspectives on the pandemic crisis were assessed. Finally, validated questionnaires, translated into French, were used to screen for anxiety/depression (Hospital Anxiety and Depression Scale [HADS$\mathrm{A} / \mathrm{D}]$ ) and post-traumatic stress disorder (PTSD checklist [PCL-5] for the Diagnostic and Statistical Manual of Mental Disorders, Fifth edition [DSM-5]). Positive screening for these mental disorders was confirmed with a score $\geq 10$ for HADS-Anxiety, $\geq 7$ for HADS-Depression [18] and $\geq 31$ for PCL-5 [19].

\section{Statistics}

Quantitative data are reported as medians and interquartile range (IQR) and were compared by non-parametric Mann-Whitney tests. Categorical data are reported as numbers (\%) and were compared by chi-square or Fisher exact tests, as appropriate. To compare initial and persistent clinical features, McNemar's test was used with Bonferroni's correction for multiple testing. Statistical analyses were carried out using GraphPad Prism 7.0. P $<0.05$ was considered statistically significant.

\section{RESULTS}

\section{Patients}

Thirty-four consecutive patients seeking help for persistent symptoms attributed to COVID19 contacted our center between June and August 2020; 30 were included in the study. Reasons for non-inclusion were refusal of physical consultation because of geographical distance $(n=3)$ or spontaneous improvement of the condition $(n=1)$. In total, $60 \%$ were women (18/30) and the median age was 40 years (IQR 35-54). The cohort included two married couples, and other patients were 
unrelated. Before the initial presentation, none of the patient reported chronic pain or use of analgesics. Other characteristics are shown in Table 1.

\section{Initial Presentation}

All patients declared a viral illness compatible with COVID-19. Initial symptoms occurred between 1 February and 9 April 2020 and were mainly characterized by fever $(60 \%)$, myalgia (76.7\%), cough (73.3\%) and anosmia (43.3\%) (Fig. 1, Table 1). Among nine patients who underwent nasopharyngeal SARS-CoV-2 RTPCR within 5 weeks of symptom onset, $55.6 \%$ $(5 / 9)$ had a positive result, and all RT-PCR tests conducted after this time were negative $(n=11)$. Seven patients visited the emergency department, and one was hospitalized in a conventional hospital unit, receiving oxygen for 7 days, without any other specific treatment. All other patients were cared for at home. A total of 13 patients were prescribed treatments: 2 received hydroxychloroquine ( $400 \mathrm{mg} /$ day), 4 prednisone ( $1 \mathrm{mg} / \mathrm{kg}$ for $5-7$ days), 8 antibiotics (azithromycin, $n=6$; amoxicillin, $n=2$ ) and 2 low-dose aspirin. The other 17 patients received only symptomatic treatment (acetaminophen).

\section{Persistent Clinical Features}

Patients were clinically evaluated after a median of 152 days (IQR 102-164) following the reported onset of initial symptoms. Seventeen $(56.7 \%)$ reported a resolution of initial symptoms after a median of 21 days (IQR 15-33), followed by a resurgence at a median of 21 days later (IQR 15-44). Conversely, the 13 other patients had no symptom-free intervals (Figure S1). Persistent symptoms had a cyclical pattern in 28 (93.3\%) patients and were mostly represented by fatigue, myalgia and thoracic oppression (Fig. 1a). Fever, shivering and cough were significantly less frequent compared with the initial presentation $(p<0.005$ for all; Fig. 1a). Fatigue was severe for most patients and rated at a median of 7 (IQR 5-8) on a 10-point scale, with pain rated at 5 (IQR 2-6).
Overall, $60 \%$ and $43.4 \%$ of patients exhibited diffuse paresthesia and burning pain later after the initial presentation (Fig. 1a). The DN4 questionnaire screening neuropathic pain was positive $(\geq 4 / 10)$ for $50 \%(15 / 30)$ of patients, and the FiRST questionnaire screening for fibromyalgia-like symptoms was positive $(\geq 5$ / 6) for 56.7\% (17/30; Figure S2).

The clinical examination, including neurologic examination, was unremarkable. At this point, no patients received corticosteroids, nonsteroidal antiinflammatory drugs or opioids. Finally, $16(53.3 \%)$ patients reported a trend toward a decrease of symptoms over time.

\section{Virologic and Specific Immunologic Evaluation}

Specific analyses related to SARS-CoV-2 infection were conducted at a median of 174 (IQR 144-215) days after symptom onset. At this time, $18(60 \%)$ and $6(20 \%)$ patients provided nasopharyngeal and stool samples for SARSCoV-2 RT-PCR; all tests were negative.

Exploration of the cellular immune response by SARS-CoV-2 IFN- $\gamma$ ELISPOT assay revealed that $15(50 \%)$ patients had a positive response to at least SARS-CoV-2 nucleocapsid and spike proteins (considered ELISPOT-positive, Fig. 2A). Among ELISPOT-positive patients, 73.3\% (11/ 15) also had a cellular response against nonstructural SARS-CoV-2 protein compared with only $1(6.7 \%)$ of ELISPOT-negative patients $(p<0.0001)$. Cellular responses to the spike protein of human coronavirus 229E and OC43 were similar in both SARS-CoV-2 ELISPOT groups (100\% in ELISPOT-positive vs. $86.7 \%$ in ELISPOT-negative and $80 \%$ in ELISPOT-positive vs. $73.3 \%$ in ELISPOT-negative, respectively).

Among patients with a negative IFN- $\gamma$ ELISPOT result, all but one had negative serology results (Fig. 2a, Table 2). This patient had an isolated positive anti-S IgG assay. Among the 15 patients with a positive IFN- $\gamma$ ELISPOT result, all had at least one positive serologic assay. In detail, 14 (93.3\%) had a positive result for antiRBD total antibodies, 11 (73.3\%) anti-RBD IgG/ IgM, 12 (80\%) anti-S IgG and 11 (73.3\%) anti-N IgG (Fig. 2a). We dichotomized the patients 
Table 1 Characteristics of the whole population, including patients with or without cellular and/or humoral immunization, and a control group of convalescent COVID-19 patients without persistent symptoms

\begin{tabular}{|c|c|c|c|c|}
\hline Characteristics, \% $(n / N)$ & $\begin{array}{l}\text { Total } \\
(N=30)\end{array}$ & $\begin{array}{l}\text { Immunized } \\
(N=15)\end{array}$ & $\begin{array}{l}\text { Non- } \\
\text { immunized } \\
(N=15)\end{array}$ & $\begin{array}{l}\text { Convalescent } \\
\text { COVID-19 } \\
(N=17)\end{array}$ \\
\hline \multicolumn{5}{|l|}{ Demographics } \\
\hline Age (median, (IQR)) & $40(35-54)$ & $40(31-58)$ & $39(35-45)$ & $40(31-45)$ \\
\hline Female sex & $60(18 / 30)$ & $46.7(7 / 15)$ & $73.3(11 / 15)$ & $76.4(13 / 17)$ \\
\hline $\begin{array}{l}\text { Close contact with confirmed COVID-19 } \\
\text { patients }\end{array}$ & $43.3(13 / 30)$ & $46.7(7 / 15)$ & $40(6 / 15)$ & $29.4(5 / 17)$ \\
\hline \multicolumn{5}{|l|}{$\begin{array}{l}\text { History of risk factors for severe SARS-CoV-2 } \\
\text { infection }\end{array}$} \\
\hline $\begin{array}{l}\text { BMI }\left(\mathrm{kg} / \mathrm{m}^{2}\right),(\text { median, }[\mathrm{IQR}]) \\
>25\end{array}$ & $\begin{array}{l}22.6 \\
{[20.4-26.3]}\end{array}$ & $\begin{array}{l}23.6[21.2-27.8] \\
40(6 / 15)\end{array}$ & $\begin{array}{l}22.4 \\
{[19.6-24.5]}\end{array}$ & $\begin{array}{l}22.0[21.0-25.0] \\
23.5(4 / 17)\end{array}$ \\
\hline$>30$ & $\begin{array}{l}30(9 / 30) \\
20(6 / 30)\end{array}$ & $26.7(4 / 15)$ & $\begin{array}{l}20(3 / 15) \\
13.3(2 / 15)\end{array}$ & 0 \\
\hline Diabetes & $10(3 / 30)$ & $13.3(2 / 15)$ & $6.7(1 / 15)$ & $\mathrm{N} / \mathrm{A}$ \\
\hline Hypertension & $3.3(1 / 15)$ & $6.7(1 / 15)$ & 0 & $\mathrm{~N} / \mathrm{A}$ \\
\hline Myocardial infarction & 0 & & & $\mathrm{~N} / \mathrm{A}$ \\
\hline Cerebrovascular event & $6.7(2 / 30)$ & $13.3(2 / 15)$ & 0 & $\mathrm{~N} / \mathrm{A}$ \\
\hline Respiratory disease & 0 & & & $\mathrm{~N} / \mathrm{A}$ \\
\hline Renal failure & 0 & & & $\mathrm{~N} / \mathrm{A}$ \\
\hline Liver failure & 0 & & & $\mathrm{~N} / \mathrm{A}$ \\
\hline Cancer & $3.3(1 / 30)$ & $6.7(1 / 15)$ & 0 & N/A \\
\hline Smoking & $43.3(13 / 30)$ & $53.3(8 / 15)$ & $33.3(5 / 15)$ & $\mathrm{N} / \mathrm{A}$ \\
\hline History & $3.3(1 / 30)$ & $0 \%$ & $6.7(1 / 15)$ & \\
\hline \multicolumn{5}{|l|}{ Active } \\
\hline \multicolumn{5}{|l|}{ Socio-economic } \\
\hline Married & 28.6 & 30.8 & 26.7 & $\mathrm{~N} / \mathrm{A}$ \\
\hline Working status: & $83.3(25 / 30)$ & $66.7(10 / 15)$ & $100(15 / 15)$ & $100(17 / 17)$ \\
\hline Employed & $10(3 / 30)$ & $20(3 / 15)$ & 0 & 0 \\
\hline Unemployed & $6.67(1 / 30)$ & $13.3(2 / 15)$ & 0 & 0 \\
\hline \multicolumn{5}{|l|}{ Retired } \\
\hline Education level: & $6.7(2 / 30)$ & $13.3(2 / 15)$ & 0 & 0 \\
\hline Below high school & $16.7(5 / 30)$ & $26.7(4 / 15)$ & $6.7(1 / 15)$ & 0 \\
\hline High school & $26.7(8 / 30)$ & $13.3(2 / 15)$ & $40(6 / 15)$ & $70.6(12 / 17)$ \\
\hline$\leq 3$ years post-high school & $50(15 / 30)$ & $46.7(7 / 15)$ & $53.3(8 / 15)$ & $29.4(5 / 17)$ \\
\hline College graduate & & & & \\
\hline
\end{tabular}

BMI, body mass index; all comparisons between immunized and non-immunized patients with persistent symptoms are non-significant 
a Clinical features in post-acute COVID patients

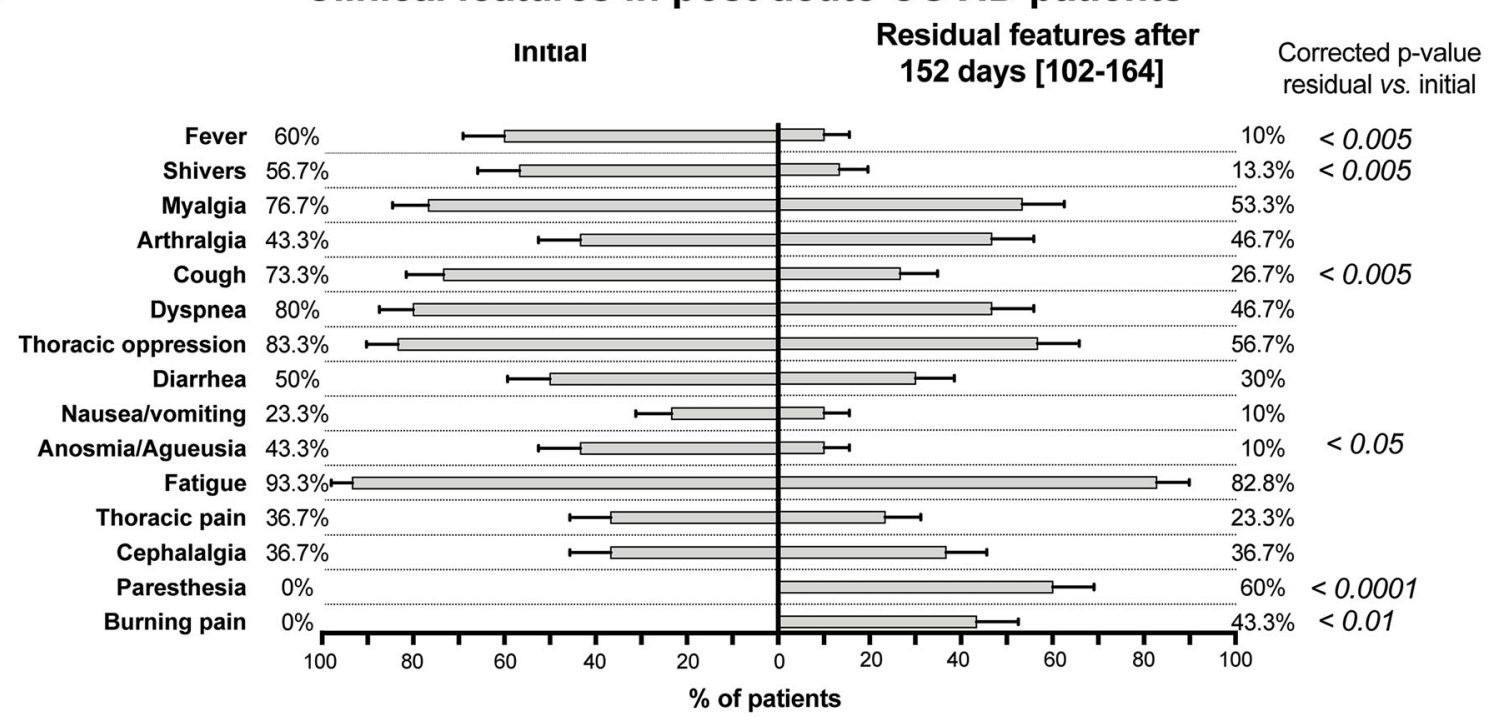

b

Clinical features in post-acute COVID patients

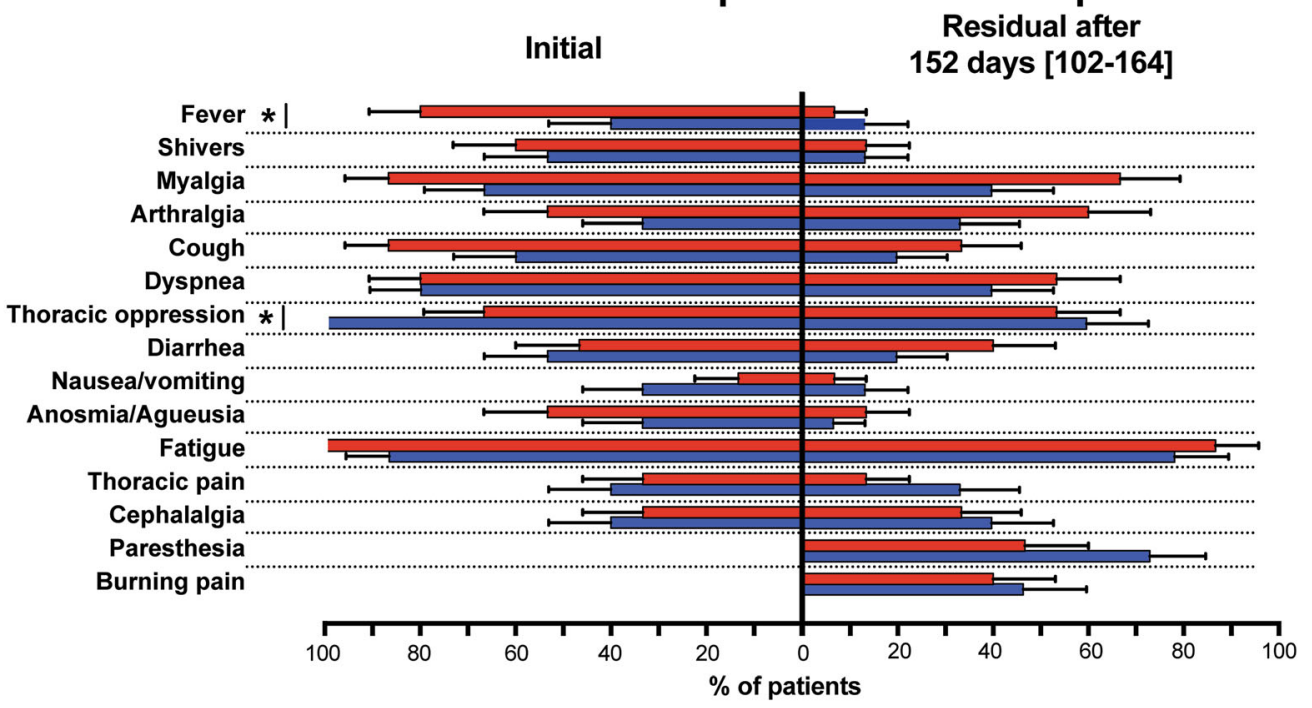

Immunized

Fig. 1 Initial and residual clinical features of patients with persistent symptoms self-attributed to COVID-19. a Residual symptoms collected at a median of 152 days (IQR 102-164) after initial presentation ( $n=30$ patients). Horizontal rectangles indicate symptom prevalence, and bars are standard error measure. $P$-values were calculated with McNemar's test with Bonferroni correction for

into an immunized group (ELISPOT-positive and at least one positive serologic assay, $n=15$ ) and a non-immunized group (ELISPOT-negative, $n=15$; Fig. 2b), who exhibited little

\section{Non-immunized}

multiple comparisons. b Comparison of initial/residual symptoms between immunized (red rectangles; positive for SARS-CoV-2 IFN- $\gamma$ ELISPOT and at least one serologic assay) and non-immunized (blue rectangles) patients. Horizontal rectangles and black bars are mean \pm standard error measure. ${ }^{*} p<0.05$ by chi-square or Fisher's exact test (if appropriate)

difference in terms of baseline characteristics and clinical features (Fig. 1b, Tables 1 and 2). Additionally, we compared patients with persistent symptoms to age-matched COVID-19 
a

Serologic assays concordance
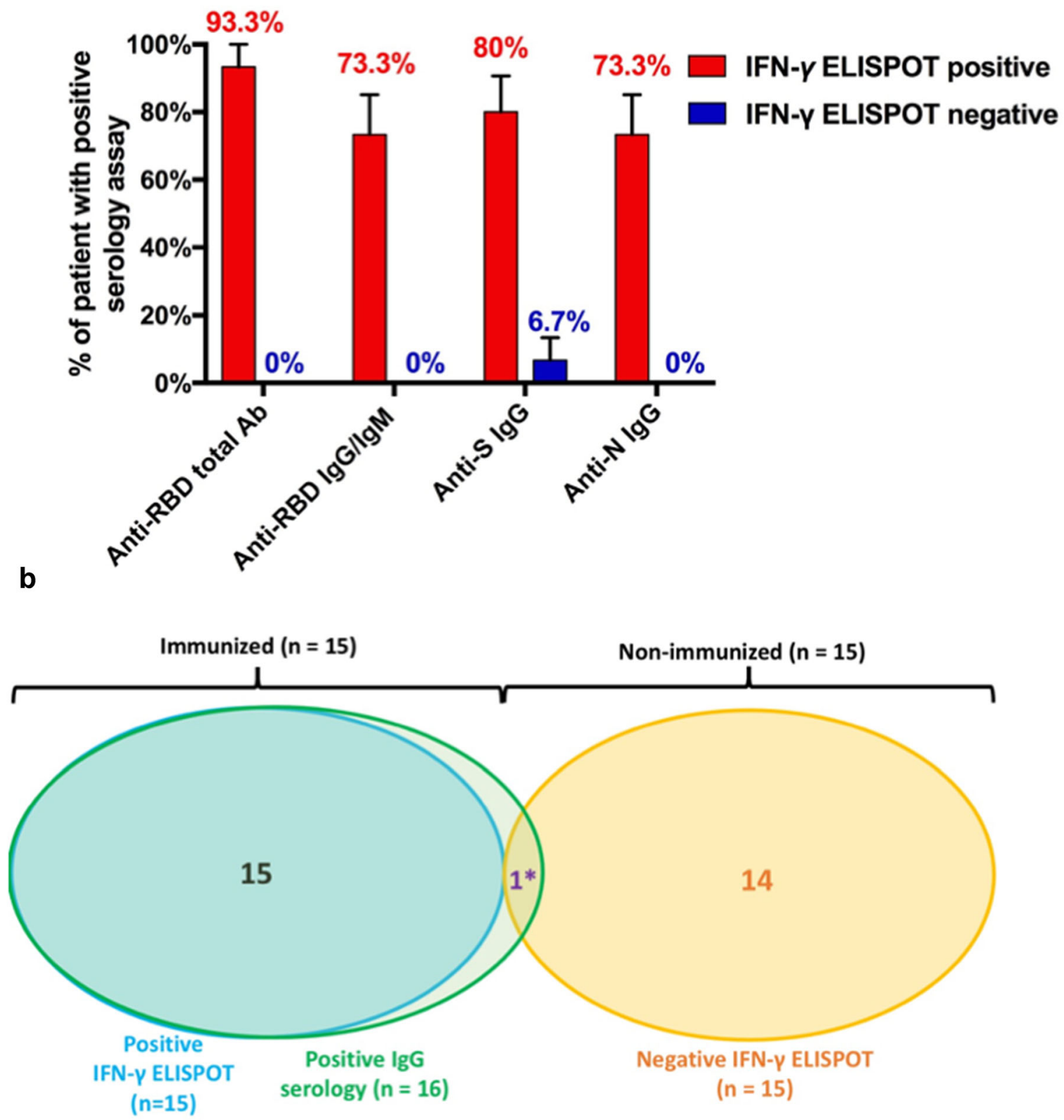

*, one isolated positive anti-S IgG assay 
4Fig. 2 Specific immunologic responses to SARS-CoV-2 in 30 patients reporting persistent symptoms self-attributed to long-COVID. a Results of SARS-CoV-2 serologic assays, according to the result of the SARS-CoV-2 interferon- $\gamma$ (IFN- $\gamma$ ) ELISPOT $(n=15 /$ group). Results for the following assays are shown: anti-RBD total antibody (Wantai total antibody); anti-RBD IgG/IgM (Biosynex BSS IgM/IgG assay); anti-S IgG (Euroimmun); anti-N IgG (Abbott Architect). Columns show the prevalence of test positivity, and black bars represent SEM. b Two patterns of patients were identified: those with objective signs of SARS-CoV-2 immunity (cellular AND humoral response, $n=15$ ) and those without $(n=15)$. Positive IgG was defined as a positive result against spike, receptor binding domain or nucleocapsid protein. ${ }^{*}$ One patient with virologically unproven initial presentation had an isolated anti-S IgG-positive assay result. IFN- $\gamma$ ELISPOT and 3 other serologic assays were negative

convalescent individuals without persistent symptoms (Table 1). Clinical features of these patients were similar to those with persistent symptoms (Table 2 ).

One ELISPOT-positive patient showed a decreasing signal for anti-N (equivocal to negative) and anti-S signal (positive to equivocal) between day 133 and day 251. One patient with negative results for both IFN- $\gamma$ ELISPOT and serology assays (conducted 140 days after symptom onset) previously had a positive nasopharyngeal RT-PCR SARS-CoV-2 result early after the first symptoms (Fig. 2b). This patient also had two negative IgG serology tests for SARS-CoV-2 at 37 and 85 days after symptom onset (Biosynex ICT and CLIA Cobas Roche, respectively).

IFN- $\alpha 2$ levels were similar for patients with persistent symptoms (regardless of their immunity status) and for convalescent COVID-19 patients without persistent symptoms (sampled $>12$ weeks after infection; Fig. 3). Other cytokine levels were low or non-detectable and were similar in immunized and non-immunized post-acute COVID-19 syndrome patients. However, we found that the level of monocyte chemotactic protein 1 (MCP-1) was significantly lower in immunized and non-immunized groups of post-acute COVID-19 syndrome patients (23.0 [11.6-33.4] and 14.1 [10.0-15.6] $\mathrm{ng} / \mathrm{ml}$, respectively) compared to the group of convalescent COVID-19 patients without persistent symptoms (33.3 [24.5-60.5] $\mathrm{ng} / \mathrm{ml}$, $p<0.05$ and $p<0.0001$, respectively; Figure S3).

\section{Biologic Evaluation}

Biologic analyses (Supplementary File 1) were conducted at the same time as the clinical evaluation (152 days [IQR 102-164] after initial presentation). Routine biologic test results were within normal limits for all but one patient (an iron-deficiency anemia that was further investigated and corrected). Values for markers of cardiac and muscle injury (troponin and creatine phosphokinase) and coagulopathy (Ddimers, fibrinogen) were normal. Serology for HIV, hepatitis $C$ virus and Lyme disease were negative for all patients.

Screening for autoimmunity revealed low (1/ $160)$ and medium (1/320 to $1 / 640)$ titers of antinuclear antibodies in 12 and 3 patients, respectively. Low to medium anti-nuclear antibody titers were numerically more prevalent in SARS-CoV-2 immunized than non-immunized patients $(66.7 \%$ vs. $33.3 \%, p=0.067)$. Screening for anti-extractable nuclear antigens, anti-double-stranded DNA, anti-citrullinated protein and anti-neutrophil cytoplasmic antibodies as well as rheumatoid factor was negative for all patients. Eight patients (4 in each immunization group) showed isolated low titers $(<3$ times normal range) of anti-cardiolipin antibodies, with no history of thrombosis (IgM for 6, IgG for 2), and one patient was positive for lupus anticoagulant. After 12 weeks, the repeat antiphospholipid antibodies testing was negative for all patients.

Taken together, these extensive biologic studies were unremarkable, and multiplex cytokine and ultra-sensitive interferon- $\alpha 2$ measurements were similar between COVID-19 long-haulers and convalescent COVID-19 individuals without persistent symptoms. Using SARS-CoV-2 serology tests and IFN- $\gamma$ ELISPOT, we found evidence of a previous SARS-CoV-2 infection in 50\% (15/30) of post-acute COVID- 
Table 2 Description of the clinical features among the included populations

\begin{tabular}{|c|c|c|c|c|}
\hline \multirow[t]{2}{*}{ Characteristics, \% (n/N) } & \multicolumn{3}{|c|}{ Post-acute COVID-19 syndrome } & \multirow{2}{*}{$\begin{array}{l}\text { Control } \\
\text { Convalescent } \\
\text { COVID-19 } \\
(N=17)\end{array}$} \\
\hline & $\begin{array}{l}\text { Total } \\
(N=30)\end{array}$ & $\begin{array}{l}\text { Immunized } \\
(N=15)\end{array}$ & $\begin{array}{l}\text { Non- } \\
\text { immunized } \\
(N=15)\end{array}$ & \\
\hline \multicolumn{5}{|l|}{ SARS-CoV-2 infection } \\
\hline Positive RT-PCR & $55.6(5 / 9)$ & $80(4 / 5)$ & $25(1 / 4)$ & $88.2(15 / 17)$ \\
\hline Hospitalization & $3.3(1 / 30)$ & $6.7(1 / 15)$ & 0 & 0 \\
\hline Ward & $3.3(1 / 30)$ & $6.7(1 / 15)$ & & \\
\hline ICU & 0 & 0 & & \\
\hline \multicolumn{5}{|l|}{ Initial clinical features } \\
\hline Fever & $60(18 / 30)$ & $80(12 / 15)$ & $40(6 / 15)$ & $76.5(13 / 17)$ \\
\hline Shivers & $56.7(17 / 30)$ & $60(9 / 15)$ & $53.3(8 / 15)$ & $47.1(8 / 17)$ \\
\hline Myalgia & $76.7(23 / 30)$ & $86.7(13 / 15)$ & $66.7(10 / 15)$ & $82.4(14 / 17)$ \\
\hline Arthralgia & $43.3(13 / 30)$ & $53.3(8 / 15)$ & $33.3(5 / 15)$ & $29.4(5 / 17)$ \\
\hline Cough & $70(21 / 30)$ & $80(12 / 15)$ & $60(9 / 15)$ & $58.8(10 / 17)$ \\
\hline Dyspnea & $80(24 / 30)$ & $80(12 / 15)$ & $80(12 / 15)$ & $58.8(10 / 17)$ \\
\hline Thoracic oppression & $83.3(25 / 30)$ & $66.7(10 / 15)^{*}$ & $100(15 / 15)^{*}$ & $82.4(15 / 17)$ \\
\hline Diarrhea & $50(15 / 30)$ & $46.6(7 / 15)$ & $53.3(8 / 15)$ & $35.3(6 / 17)$ \\
\hline Nausea/vomiting & $23.3(7 / 30)$ & $13.3(2 / 15)$ & $33.3(5 / 15)$ & $23.5(4 / 17)$ \\
\hline Anosmia/agueusia & $43.3(13 / 30)$ & $53.3(8 / 15)$ & $33.3(5 / 15)$ & $58.8(10 / 17)$ \\
\hline Fatigue & $93.3(28 / 30)$ & $100(15 / 15)$ & $86.7(13 / 15)$ & $94.1(16 / 17)$ \\
\hline Thoracic pain & $36.7(11 / 30)$ & $33.3(5 / 15)$ & $40(6 / 15)$ & $33.3(5 / 17)$ \\
\hline Cephalalgia & $36.7(11 / 30)$ & $33.3(5 / 15)$ & $40(6 / 15)$ & $76.5^{\# \#}(13 / 17)$ \\
\hline Paresthesia & 0 & 0 & 0 & 0 \\
\hline Burning pain & 0 & 0 & 0 & 0 \\
\hline \multicolumn{5}{|l|}{ Immuno-virologic testing } \\
\hline Time between symptom onset and blood test, median (IQR) & $\begin{array}{l}174 \\
\quad[144-214]\end{array}$ & $151[144-190]$ & $\begin{array}{l}187 \\
\quad[145-213]\end{array}$ & $96[94-110]$ \\
\hline Positive SARS-CoV-2 serology (according to at least one test) & $53.3(16 / 30)$ & $100(15 / 15)^{* * *}$ & $6.7(1 / 15)^{* * *}$ & $100(17 / 17)^{\# \# \#}$ \\
\hline Positive SARS-CoV-2 ELISPOT & $50(15 / 30)$ & $100(15 / 15)^{* * *}$ & $0^{* * *}$ & $100(15 / 15)^{\# \# \#}$ \\
\hline \multicolumn{5}{|l|}{ Persistent clinical features } \\
\hline Cyclical pattern of symptoms & $93.3(28 / 30)$ & $100(15 / 15)$ & $86.7(13 / 15)$ & N/A \\
\hline Symptom-free interval between initial presentation and persistent symptoms, if & $56.6(17 / 30)$ & $60(9 / 15)$ & $53.3(8 / 15)$ & N/A \\
\hline any $(\mathrm{n} / \mathrm{N})$, in days (median [IQR]) & $26[15-44]$ & $19[16-61]$ & $26[15-34]$ & \\
\hline Fever & $10 \%(3 / 30)$ & $6.6(1 / 15)$ & $13.3(2 / 15)$ & N/A \\
\hline Shivers & $13.3(4 / 30)$ & $13.3(2 / 15)$ & $13.3(2 / 15)$ & N/A \\
\hline Myalgia & $53.3(16 / 30)$ & $66.7(10 / 15)$ & $40(6 / 15)$ & N/A \\
\hline Arthralgia & $46.6(14 / 30)$ & $60(9 / 15)$ & $33.3(5 / 15)$ & N/A \\
\hline
\end{tabular}


Table 2 continued

\begin{tabular}{|c|c|c|c|c|}
\hline \multirow[t]{2}{*}{ Characteristics, \% (n/N) } & \multicolumn{3}{|c|}{ Post-acute COVID-19 syndrome } & \multirow{2}{*}{$\begin{array}{l}\text { Control } \\
\text { Convalescent COVID-19 } \\
(N=17)\end{array}$} \\
\hline & $\begin{array}{l}\text { Total } \\
(N=30)\end{array}$ & Immunized $(N=15)$ & $\begin{array}{l}\text { Non- immunized } \\
(N=15)\end{array}$ & \\
\hline Cough & $26.7(8 / 30)$ & $33.3(5 / 15)$ & $20(3 / 15)$ & N/A \\
\hline Dyspnea & $46.7(14 / 30)$ & $53.3(8 / 15)$ & $40(6 / 15)$ & N/A \\
\hline Thoracic oppression & $56.7(17 / 30)$ & $53.3(8 / 15)$ & $60(9 / 15)$ & N/A \\
\hline Diarrhea & $30(9 / 30)$ & $40(6 / 15)$ & $20(3 / 15)$ & N/A \\
\hline Nausea/vomiting & $10(3 / 30)$ & $6.7(1 / 15)$ & $13.3(2 / 15)$ & N/A \\
\hline Anosmia/agueusia & $10(3 / 30)$ & $13.3(2 / 15)$ & $6.7(1 / 15)$ & N/A \\
\hline Fatigue & $83.3(25 / 30)$ & $86.7(13 / 15)$ & $80(12 / 15)$ & N/A \\
\hline Thoracic pain & $23.3(7 / 30)$ & $13.3(2 / 15)$ & $33.3(5 / 15)$ & N/A \\
\hline Cephalalgia & $36.7(11 / 30)$ & $33.3(5 / 15)$ & $40(6 / 15)$ & N/A \\
\hline Paresthesia & $60(18 / 30)$ & $46.7(7 / 15)$ & $73.3(11 / 15)$ & N/A \\
\hline Burning pain & $43.3(13 / 30)$ & $40(6 / 15)$ & $46.7(7 / 15)$ & $\mathrm{N} / \mathrm{A}$ \\
\hline
\end{tabular}

${ }^{*} p<0.05,{ }^{* * *} p<0.001$ using chi $^{2}$ or Fisher's exact test when comparing the immunized and non-immunized patients with persistent symptoms $\# p<0.05 ; \# \#>0.01, \# \# p<0.001$ using chi $^{2}$ or Fisher's exact test when comparing the patient without persistent symptoms attributed to SARS-CoV-2 $(n=30)$ and convalescent COVID-19 patients without persistent symptoms $(n=17)$. N/A, non-assessable

19 syndrome patients, with evidence of a lack of immune response, or a waning immune response, in two patients.

\section{Psychologic Evaluation}

Psychologic evaluation was carried out by a phone interview with a clinical psychologist, at median 224 [202-238] days after initial symptom onset. In all, $10 \%(3 / 30)$ and $26.7 \%(8 / 30)$ of patients had a history of depression and anxiety disorders, respectively. Sleep issues were reported by $23(73.3 \%)$ patients, and 4 (13.3\%) had started an anxiolytic prescription (Table S1); 5 (16.7\%) and 7 (23.3\%) reported loss of employment and financial difficulties.

HADS screening for anxiety and depression was positive for $11(36.7 \%)$ and 13 (43.3\%) of patients, respectively (Table S1). Using the PCL5 questionnaire, nine (30\%) patients had scores compatible with PTSD (Fig. 4). These values did not differ between immunized and non-imunized groups of post-acute COVID-19 syndrome patients. Several components of the SF-36 scale, physical limitations, energy and pain, were severely affected, equally with no significant difference between patients immunized, or not, for SARS-CoV-2 (Figure S4). Family, friends and colleagues were a major source of support for 20 (66.7\%), $18(60 \%)$ and 11/25 (44\%) patients, respectively. Conversely, 7 (23.3\%) patients felt that physicians provided a significant level of support.

\section{DISCUSSION}

In this study, we included 30 consecutive patients seeking medical help for persistent symptoms (median of 6 months) self-attributed to COVID-19. We identified two clinically comparable groups of post-acute COVID-19 syndrome patients: those with and those without SARS-CoV-2 immunity. In 93\% of patients, persistent symptoms had a cyclical pattern and were mostly represented by fatigue, thoracic oppression, myalgia, paresthesia and burning pain, which agrees with the literature [20, 21]. We failed to find a significant difference in 


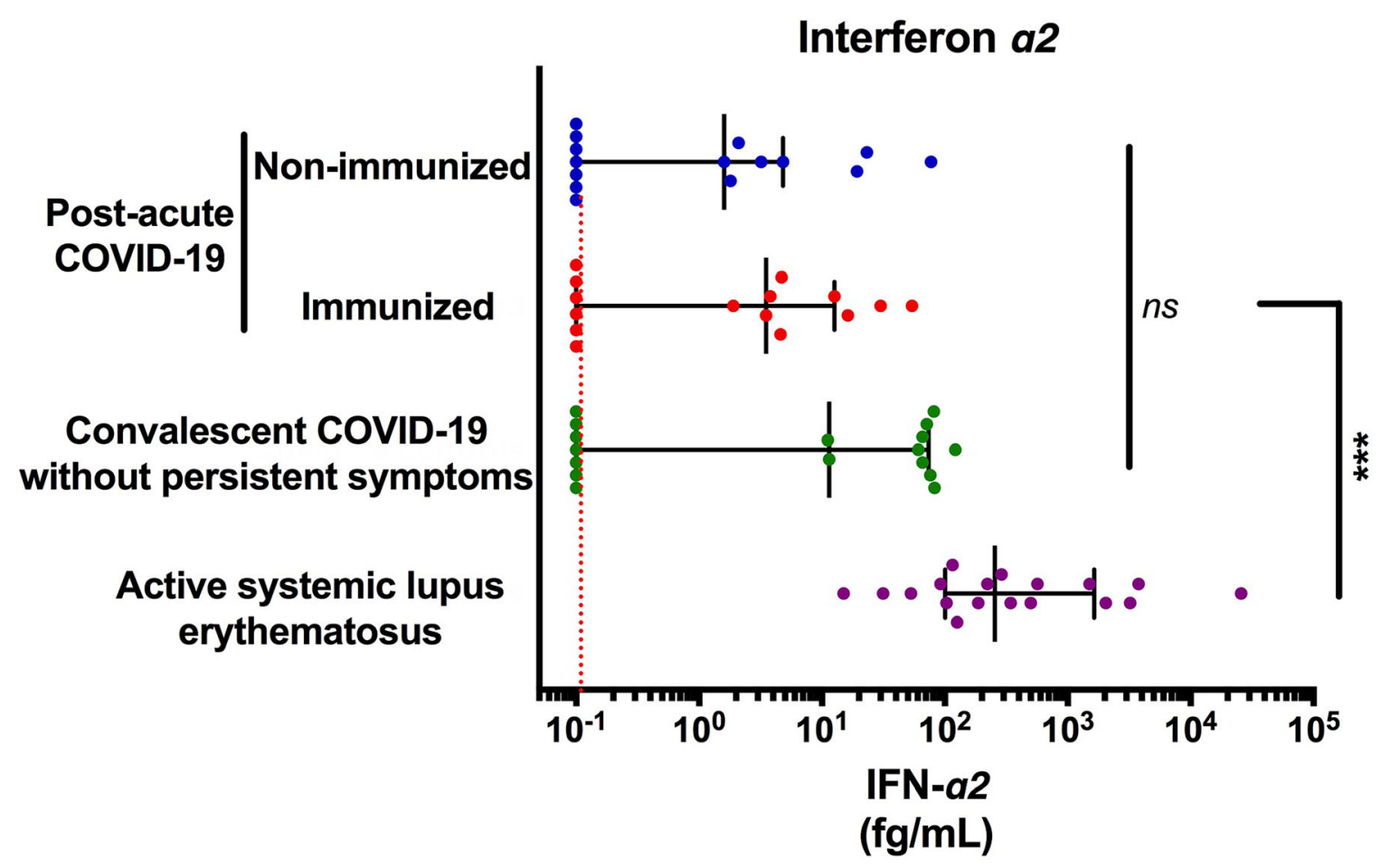

Fig. 3 Normal levels of IFN- $\alpha 2$ for patients with persistent symptoms attributed to COVID-19 compared to convalescent COVID-19 individuals. Ultra-sensitive IFN- $\alpha 2$ levels were measured by using Single Molecule Array (SIMOA) in patients with persistent symptoms selfattributed to COVID-19 (post-acute COVID-19 syndrome) whether they were immunized against SARS-CoV2 (positive for SARS-CoV-2 IFN- $\gamma$ ELISPOT and at least one serologic assay, $n=15)$ or non-immunized $(n=15)$. As a comparison, results from individuals with confirmed

persistent clinical features between anti-SARSCoV-2 immunized and non-immunized patients (Fig. 1b), and both groups were characterized by similar pain/fatigue indexes, quality of life, cytokines levels and psychologic burden. Additionally, patients with post-acute COVID-19 syndrome had similar initial clinical features and interferon-a2 levels compared to COVID-19-convalescent individuals who had experienced no persistent symptoms.

Unexpectedly, only half of post-acute COVID-19 syndrome patients had cellular (IFN$\gamma$ ELISPOT-based) and humoral immunity for SARS-CoV-2 (Fig. 2b). There are three possible (and non-exclusive) explanations for this result.
COVID-19 (serology and IFN- $\gamma$ positive) without persistent symptoms (sampled at least 12 weeks after infection; $n=17$ ) and patients with active systemic lupus erythematosus $(n=18)$ are shown. Each point corresponds to a single patient; the central bar shows the median with interquartile ranges. The red dotted line shows the lower limit of detection. Ns, non-significant; ${ }^{* * *} p<0.001$ versus all other groups by non-parametric Kruskal-Wallis test with Dunn's correction for multiple testing

First, some patients may have been infected with SARS-CoV-2, but not developed detectable immunity. As already described [22], one of our patients with RT-PCR-proven SARSCoV-2 infection had a negative SARS-CoV-2 serology result on several occasions $(36,85$ and 140 days after initial symptoms) as well as a negative IFN- $\gamma$ ELISPOT (after 140 days). Second, immunity may have developed in some patients but subsequently waned over time, although such reports are discordant in the literature $[23,24]$. In our cohort, one patient with RT-PCR/ELISPOT-confirmed infection presented waning IgG serology levels between days 133 and 251, both on anti-N (from equivocal to 


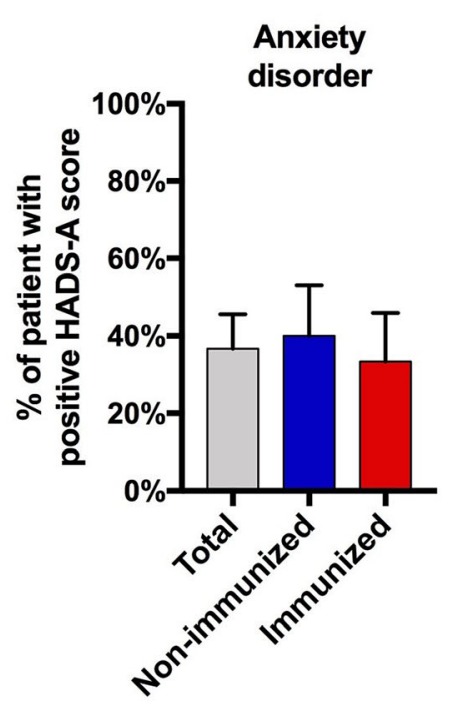

Fig. 4 Prevalence of anxiety/depression disorders and post-traumatic stress syndrome in patients seeking medical help for persistent symptoms self-attributed to COVID19. Total population $(n=30$, gray bar $)$, patients

negative) and anti-S (from positive to equivocal) ELISA. Additionally, patients with cellular immunity might present negative SARS-CoV-2 serology tests, particularly with regard to anti-N IgG (Fig. 2a) [25]. Finally, some patients may have presented a non-specific viral illness and subsequent symptoms, which were falsely attributed to SARS-CoV-2. The first pandemic wave was a period of high anxiety for many people and may have exacerbated pre-existing psychologic conditions and induced a nocebo effect in some patients [26]. Although it could be argued that some of these patients may not, in fact, have initially developed COVID-19, we believe it is important to study these patients without any preconceived ideas, since they may represent a significant proportion of post-acute COVID-19 syndrome patients, at least in our cohort.

The high prevalence of probable anxiety and depressive disorders $(36.7 \%$ and $43.3 \%$ respectively) is striking. A cohort study in the UK revealed that the incidence of probable anxiety and depressive disorders in the general population was $24 \%$ and $18.1 \%$, respectively, during the pandemic [27]. A history of reported COVID-19 was associated with increased risk anxiety and depression. We also highlighted a high rate of probable PTSD (33.3\%), which is similar to the $30.8 \%$ prevalence observed in a US study [28]. PTSD reflects the hardship of the patient's experience with COVID-19. The persistence of physical symptoms (related or not to COVID-19) is likely associated with the psychologic burden of the pandemic, synergistically contributing to the emergence of the postacute COVID-19 syndrome and may explain the high prevalence of psychologic disorders in our cohort.

Post-acute COVID-19 syndrome is an ill-defined condition characterized by symptoms persisting for at least 4 to 12 weeks after SARSCoV-2 infection, depending on the study $[11,13]$. It is crucial to exclude any alternative diagnoses before diagnosis of post-acute COVID-19 syndrome, especially as this latter does not necessarily require evidence of SARSCoV-2 immunity $[13,20]$. Deep phenotyping of our patients did not lead to alternative diagnoses for any patient. Although most authors studying post-acute COVID-19 syndrome focused on patients hospitalized with COVID19 [29], a recent study including 958 patients with mild COVID-19 found persistent 
symptoms after 4 months in $27.8 \%$ of cases. This study, together with ours, suggests that post-acute COVID-19 syndrome may follow a mild initial infection in a high number of cases. The pathophysiologic mechanisms underlying post-acute COVID-19 syndrome are likely to be multifactorial in such patients, and physical deconditioning [30], psychologic disorders [31], viral encephalitis [7], dysautonomia [32] and autoimmunity are plausible suspects which could co-exist. Indeed, autoimmunity has been documented following COVID-19 [10, 33]. However, viral-induced autoimmunity is likely transient, as demonstrated by the negative repeat screening for antiphospholipid antibodies in eight initially positive patients. We did not find a significant increase in IFN- $\alpha 2$ or other proinflammatory cytokines between post-acute COVID-19 syndrome patients and convalescent individuals without persistent symptoms. The only difference was a significantly lower level of MCP-1 in post-acute COVID-19 syndrome patients, for which higher levels have been associated with severe disease and blood-brain barrier permeability $[34,35]$. Further studies will be needed to explore other potential mechanisms at play in such patients (e.g., dysautonomia, neurologic studies).

This study has several limitations. First, our recruitment was limited to 30 consecutive patients, which may not allow capturing the complexity of patients with post-acute COVID19 syndrome and thus limits its generalizability. However, this relatively small cohort allowed for an extensive multimodal evaluation without significant missing data. Additionally, the consecutive recruitment method limited potential selection bias. Given our study design, however, we cannot infer the prevalence of post-acute COVID-19 syndrome, which has been evaluated at $10 \%$ in the UK [11]. Second, only nine patients had a RT-PCR test in our cohort in the 5 weeks after symptom onset because RT-PCR tests were not readily available for mild disease during the first epidemic wave in France. To investigate for viral persistence at the time of clinical evaluation, we asked patients to make an appointment for nasopharyngeal and stool RT-PCR tests, which were only carried out by $60 \%$ and $20 \%$ of patients, respectively. These tests were negative and argue against viral persistence in clinically accessible sites. Finally, we cannot exclude that our design implied a selection bias of patients with psychologic distress. However, our study did allow to accurately and thoroughly evaluate multiple biologic and clinical characteristics of patients seeking medical advice for persistent symptoms self-attributed to post-acute COVID-19 syndrome and will therefore be of use for clinicians who may be under-prepared to answer these patients' questions. Although no treatment has been approved for post-acute COVID-19 syndrome, more than half of our patients reported a spontaneous and gradual improvement of symptoms over time, which provides a window of hope for patients suffering from this disorder.

\section{CONCLUSION}

To conclude, our study sheds light on the burden experienced by patients reporting longterm symptoms self-attributed to COVID-19 as well as some of the mechanisms at play. A better recognition and understanding of post-acute COVID-19 syndrome will help healthcare providers to better care for these patients.

\section{ACKNOWLEDGEMENTS}

We thank all patients who agreed to take part in the study. We thank Valle Meyer for her precious help in planning the study and patient communication. We also thank COVID-HUS and Strasbourg University Hospital for their support.

Funding. No funding or sponsorship was received for this study or publication of this article. The Journal's Rapid Service Fee was funded by the authors.

Editorial Assistance . Editorial assistance in the production of this manuscript was provided by Dr. Kate Dunning (Hôpitaux Universitaires de Strasbourg). 
Authorship. All named authors meet the International Committee of Medical Journal Editors (ICMJE) criteria for authorship for this article, take responsibility for the integrity of the work as a whole, and have given their approval for this version to be published.

Authorship Contributions. Marc Scherlinger, Renaud Felten, Jean Sibilia, Emmanuel Chatelus and Luc Pijnenburg recruited and evaluated clinically the patients. Floriane Gallais, Charlotte Nazon and Samira Fafi-Kremer conducted the virologic evaluations. Amaury Mengin, Adrien Gas and Pierre Vidailhet participated in the psychiatric evaluation. Rachel Arnould-Michel conducted the psychologic evaluation. Sabrina Bibi-Triki, Raphael Carapito, Sophie Trouillet-Assant, Magali Perre, Alexandre Belot and Seiamak Bahram conducted the immunologic assays (cytokines). Laurant Arnaud, Jacques Eric-Gottenberg helped in the design of the study and critically reviewed the manuscript. Marc Scherlinger, Renaud Felten, Floriane Gallais, Samira FafiKramer and Jean Sibilia wrote the manuscript. Marc Scherlinger, Renaud Felten and Floriane Gallais are co-first authors. Samira Fafi-Kramer and Jean Sibilia are co-last authors.

Disclosures. Samira Fafi-Kremer has received speaking fees from BMS and Roche.

Compliance with Ethics Guidelines. Patients recruited in the present study provided written consent to participate. The study was conducted in accordance with the Helsinki Declaration of 1964 and its later amendments. This study is part of the "COVID-HUS" study, which was approved by the Ethics Committee of the University Hospital of Strasbourg (NCE-2020-51). Individuals with a history of COVID-19 without persistent symptoms, were recruited as part of the Study "SeroCOVHUS", ClinicalTrials.gov Identifier: NCT04441684, Ethical Committee authorization from SUD MEDITERRANEE III, Number 2020.04.15 bis_ 20.04.10.66856).

Data Availability. Data are available upon request to the corresponding authors.
Open Access. This article is licensed under a Creative Commons Attribution-NonCommercial 4.0 International License, which permits any non-commercial use, sharing, adaptation, distribution and reproduction in any medium or format, as long as you give appropriate credit to the original author(s) and the source, provide a link to the Creative Commons licence, and indicate if changes were made. The images or other third party material in this article are included in the article's Creative Commons licence, unless indicated otherwise in a credit line to the material. If material is not included in the article's Creative Commons licence and your intended use is not permitted by statutory regulation or exceeds the permitted use, you will need to obtain permission directly from the copyright holder. To view a copy of this licence, visit http:// creativecommons.org/licenses/by-nc/4.0/.

\section{REFERENCES}

1. Carfì A, Bernabei R, Landi F, for the Gemelli Against COVID-19 Post-Acute Care Study Group. Persistent symptoms in patients after acute COVID-19. JAMA. 2020;324(6):603.

2. Living with Covid19 [Internet]. National Institute for Health Research; 2020 Oct [cited 2020 Nov 13]. Available from: https://evidence.nihr.ac.uk/ themedreview/living-with-covid19/

3. Covid Long Haulers Describe the Devastating Aftereffects of the Disease. Bloomberg.com [Internet]. 2020 Nov 9 [cited 2020 Dec 5]; Available from: https://www.bloomberg.com/news/features/202011-09/coronavirus-long-haulers-tell-us-theirsymptoms-and-the-aftereffects-of-disease

4. Long Covid: 'Is this now me forever?' [Internet]. the Guardian. 2020 [cited 2020 Dec 5]. Available from: http://www.theguardian.com/world/2020/nov/29/ eleanor-morgan-is-still-struggling-with-long-covidmonths-after-catching-the-virus

5. Martinez MW, Tucker AM, Bloom OJ, Green G, DiFiori JP, Solomon G, et al. Prevalence of Inflammatory Heart Disease Among Professional Athletes With Prior COVID-19 Infection Who Received Systematic Return-to-Play Cardiac Screening. JAMA Cardiol [Internet]. 2021 Mar 4 [cited 2021 Jun 1]; Available from: https://jamanetwork.com/journals/ jamacardiology/fullarticle/2777308 
6. Wu X, Liu X, Zhou Y, Yu H, Li R, Zhan Q, et al. 3-month, 6-month, 9-month, and 12-month respiratory outcomes in patients following COVID-19related hospitalisation: a prospective study. Lancet Respir Med [Internet]. 2021 May 5 [cited 2021 Jun 1];0(0). Available from: https://www.thelancet. com/journals/lanres/article/PIIS2213-2600(21)0017 4-0/abstract

7. Pero A, Ng S, Cai D. COVID-19: a perspective from clinical neurology and neuroscience. Neuroscientist. 2020;26(5-6):387-91.

8. Chopra V, Flanders SA, O'Malley M, Malani AN, Prescott HC. Sixty-day outcomes among patients hospitalized with COVID-19. Ann Intern Med. 2020;174(4):576-8.

9. Huang C, Huang L, Wang Y, Li X, Ren L, Gu X, et al. 6-month consequences of COVID-19 in patients discharged from hospital: a cohort study. The Lancet. $2021 ; 397(10270): 220-32$.

10. Ramos-Casals M, Brito-Zerón P, Mariette X. Systemic and organ-specific immune-related manifestations of COVID-19. Nat Rev Rheumatol. $2021 ; 17(6): 315-32$.

11. Prevalence of long COVID symptoms and COVID19 complications - Office for National Statistics [Internet]. [cited 2020 Dec 16]. Available from: https://www.ons.gov.uk/peoplepopulationandcom munity/healthandsocialcare/healthandlifeexpectan cies/datasets/prevalenceoflongcovidsymptomsandc ovid19 complications

12. Yelin D, Wirtheim E, Vetter P, Kalil AC, Bruchfeld J, Runold $M$, et al. Long-term consequences of COVID-19: research needs. Lancet Infect Dis. 2020;20(10):1115-7.

13. Raveendran AV. Long COVID-19: Challenges in the diagnosis and proposed diagnostic criteria. Diabetes Metab Syndr Clin Res Rev. 2021;15(1):145-6.

14. Salje H, Kiem CT, Lefrancq N, Courtejoie N, Bosetti $\mathrm{P}$, Paireau J, et al. Estimating the burden of SARSCoV-2 in France. Science. 2020;369(6500):208-11.

15. Cruccu G, Sommer C, Anand P, Attal N, Baron R, Garcia-Larrea L, et al. EFNS guidelines on neuropathic pain assessment: revised 2009: Neuropathic pain assessment. Eur J Neurol. 2010;17(8):1010-8.

16. Perrot S, Bouhassira D, Fermanian J. Development and validation of the Fibromyalgia Rapid Screening Tool (FiRST): Pain. 2010;150(2):250-6.

17. Rodero MP, Decalf J, Bondet V, Hunt D, Rice GI, Werneke $S$, et al. Detection of interferon alpha protein reveals differential levels and cellular sources in disease. J Exp Med. 2017;214(5):1547-55.
18. Roberge P. A psychometric evaluation of the French Canadian version of the Hospital Anxiety and Depression Scale in a large primary care population. J Affect Disord. 2013;9.

19. Bovin MJ, Marx BP, Weathers FW, Gallagher MW, Rodriguez P, Schnurr PP, et al. Psychometric properties of the PTSD Checklist for Diagnostic and Statistical Manual of Mental Disorders-Fifth Edition (PCL-5) in veterans. Psychol Assess. 2016;28(11): 1379-91.

20. Greenhalgh T, Knight M, A'Court C, Buxton M, Husain L. Management of post-acute covid-19 in primary care. BMJ. 2020;m3026.

21. Ladds E, Rushforth A, Wieringa S, Taylor S, Rayner C, Husain L, et al. Persistent symptoms after Covid19: qualitative study of 114 "long Covid" patients and draft quality principles for services. BMC Health Serv Res. 2020;20(1):1144.

22. Schwarzkopf S, Krawczyk A, Knop D, Klump H, Heinold A, Heinemann FM, et al. Cellular Immunity in COVID-19 Convalescents with PCR-Confirmed Infection but with Undetectable SARS-CoV2-Specific IgG - Volume 27, Number 1-January 2021 - Emerging Infectious Diseases journal - CDC. [cited 2021 Jun 1]; Available from: https://wwwnc. cdc.gov/eid/article/27/1/20-3772_article

23. Ibarrondo FJ, Fulcher JA, Goodman-Meza D, Elliott J, Hofmann C, Hausner MA, et al. Rapid decay of anti-SARS-CoV-2 antibodies in persons with mild Covid-19. N Engl J Med. 2020;383(11):1085-7.

24. Tonn T, Corman VM, Johnsen M, Richter A, Rodionov RN, Drosten C, et al. Stability and neutralising capacity of SARS-CoV-2-specific antibodies in convalescent plasma. Lancet Microbe. 2020;1(2):e63.

25. Gallais F, Velay A, Nazon C, Wendling M-J, Partisani $\mathrm{M}$, Sibilia $\mathrm{J}$, et al. Intrafamilial exposure to SARS-CoV-2 associated with cellular immune response without seroconversion, France. Emerg Infect Dis. 2021;27(1):113-21.

26. Amanzio M, Howick J, Bartoli M, Cipriani GE, Kong J. How do nocebo phenomena provide a theoretical framework for the COVID-19 pandemic? Front Psychol. 2020;11:589884.

27. Kwong ASF, Pearson RM, Adams MJ, Northstone K, Tilling K, Smith D, et al. Mental health before and during COVID-19 in two longitudinal UK population cohorts. Br J Psychiatry. 2020;24:1-27.

28. Liu CH, Zhang E, Wong GTF, Hyun S, Hahm H "Chris". Factors associated with depression, anxiety, and PTSD symptomatology during the COVID19 pandemic: Clinical implications for U.S. young 
adult mental health. Psychiatry Res. 2020;290: 113172.

29. Ghosn J, Piroth L, Epaulard O, Turnier PL, Mentré F, Bachelet D, et al. Persistent COVID-19 symptoms are highly prevalent 6 months after hospitalization: results from a large prospective cohort. Clin Microbiol Infect [Internet]. 2021 May 10 [cited 2021 May 20];0(0). Available from: https://www. clinicalmicrobiologyandinfection.com/article/ S1198-743X(21)00147-6/abstract

30. Covid-19 will be followed by a deconditioning pandemic [Internet]. The BMJ. 2020 [cited 2021 Jan 3]. Available from: https://blogs.bmj.com/bmj/ 2020/06/15/covid-19-will-be-followed-by-adeconditioning-pandemic/

31. Butler M, Pollak TA, Rooney AG, Michael BD, Nicholson TR. Neuropsychiatric complications of covid-19. BMJ. 2020 Oct 13;371:m3871.
32. Dani M, Dirksen A, Taraborrelli P, Torocastro M, Panagopoulos D, Sutton R, et al. Autonomic dysfunction in 'long COVID': rationale, physiology and management strategies. Clin Med Lond Engl. 2021;21(1):e63-7.

33. Wang EY, Mao T, Klein J, Dai Y, Huck JD, Jaycox JR, et al. Diverse functional autoantibodies in patients with COVID-19. Nature. 2021;19:1-9.

34. Jøntvedt Jørgensen M, Holter JC, Christensen EE, Schjalm C, Tonby K, Pischke SE, et al. Increased interleukin- 6 and macrophage chemoattractant protein-1 are associated with respiratory failure in COVID-19. Sci Rep. 2020;10(1):21697.

35. Stamatovic SM, Shakui P, Keep RF, Moore BB, Kunkel SL, Van Rooijen $\mathrm{N}$, et al. Monocyte chemoattractant protein-1 regulation of bloodbrain barrier permeability. J Cereb Blood Flow Metab Off J Int Soc Cereb Blood Flow Metab. 2005;25(5):593-606. 